\title{
ON THE STABILITY OF NONLOCAL BOUNDARY VALUE PROBLEM FOR A THIRD ORDER PDE
}

\author{
Kheireddine Belakroum \\ Department of Mathematics \\ Mentouri Brothers University \\ Constantine - 25000, ALGERIA
}

\begin{abstract}
In this paper, the nonlocal boundary value problem for third order partial differential equations in a Hilbert space with a self-adjoint positive definite operator is studied. The main theorem on stability of this problem is established. In practice, stability estimates for the solution of two problems for third order partial differential equations are obtained.
\end{abstract}

AMS Subject Classification: 35G15, 47A62

Key Words: stability; Hilbert space; third order partial differential equation; self-adjoint positive definite operator

\section{Introduction}

Boundary value problems for third order partial differential equations have been a major research area in thermal conductivity [18], microscale heat transfer [19] and modern physics [15].

Many applied problems in fluid mechanics, other areas of physics and mathematical biology were formulated into nonlocal mathematical models. The wellposedness of various nonlocal boundary value problems for partial differential and difference equations have been studied extensively by many researchers (see [1], [2], [3], [4], [5], [6], [7], [8], [9], [10], [11], [12], [14], [16], [17] and the references given therein).

Received: December 24, 2020

(C) 2021 Academic Publications 
In the present paper we consider the nonlocal boundary value problem for the third order partial differential equation

$$
\left\{\begin{array}{l}
\frac{d^{3} u(t)}{d t^{3}}+\mu \frac{d^{2} u(t)}{d t^{2}}+A \frac{d u(t)}{d t}=f(t), \quad 0<t<1, \quad \mu>0, \\
u(0)=\gamma u(\lambda)+\varphi, \quad u^{\prime}(0)=\alpha u^{\prime}(\lambda)+\psi, \quad|\gamma|<1, \\
u^{\prime \prime}(0)=\beta u^{\prime \prime}(\lambda)+\xi, \quad 0<\lambda \leq 1,
\end{array}\right.
$$

in a Hilbert space $H$ with a self-adjoint positive definite operator $A \geq \delta I$, where $\delta>0$.

We are interested in studying the stability of solutions of nonlocal boundary value problem (1). A function $u(t)$ is a solution of problem (1) if the following conditions are satisfied:

(i) $u(t)$ is thrice continuously differentiable on the interval $(0,1)$ and twice continuously differentiable on the segment $[0,1]$. The derivatives at the end points of the segment are understood as the appropriate unilateral derivatives.

(ii) The element $u^{\prime}(t)$ belongs to $D(A), \forall t \in[0,1]$, and the function $A u^{\prime}(t)$ is continuous on $[0,1]$.

(iii) $u(t)$ satisfies the equation and boundary nonlocal conditions (1).

Throughout this paper, $\{C(t), t \geq 0\}$ is an operator function, $C(t)=$ $\cos \left(t B^{\frac{1}{2}}\right)$ defined by the formula

$$
C(t)=\frac{e^{i t B^{\frac{1}{2}}}+e^{-i t B^{\frac{1}{2}}}}{2},
$$

then from the following of operator function $S(t)=B^{-\frac{1}{2}} \sin \left(t B^{\frac{1}{2}}\right), S(t)=$ $\int_{0}^{t} C(p) d p$, it follows that

$$
S(t)=B^{-\frac{1}{2}} \frac{e^{i t B^{\frac{1}{2}}}-e^{-i t B^{\frac{1}{2}}}}{2 i} .
$$

Here $B=A-\frac{\mu^{2}}{4} I$, for the theory of the cosine operator function, we refer to [13].

The paper is organized as follows. In Section 2, the main theorem on stability of problem (1) is proved. In Section 3, the stability estimates for the solution of two problems for partial differential equation of a third order in $t$ are obtained. 


\section{Main Theorem on Stability}

Let us give some lemmas that will be further needed.

Lemma 1. ([10], [13]) Assume that $\delta>\frac{\mu^{2}}{4}$ Then, for $t \geq 0$ the following estimates hold:

$$
\left\|\exp \left\{ \pm i t B^{\frac{1}{2}}\right\}\right\|_{H \rightarrow H} \leq 1, \quad\|C(t)\|_{H \rightarrow H} \leq 1,\left\|B^{\frac{1}{2}} S(t)\right\|_{H \rightarrow H} \leq 1
$$

Lemma 2. ([4]) Assume that

$$
\left|1+\alpha \beta e^{-\mu \lambda}\right|>\left(|\alpha+\beta|+\frac{\frac{\mu}{2}|\alpha-\beta|}{\sqrt{\delta-\frac{\mu^{2}}{4}}}\right) e^{-\frac{\mu \lambda}{2}} .
$$

Then the operator $\Delta$ defined by the following formula

$$
\Delta=\left(1+\alpha \beta e^{-\mu \lambda}\right) I-\left[(\alpha+\beta) c(\lambda)+\frac{\mu}{2}(\alpha-\beta) s(\lambda)\right] e^{-\frac{\mu \lambda}{2}}
$$

has a bounded inverse $P=\Delta^{-1}$ and the following estimates hold:

$$
\|P\|_{H \rightarrow H} \leq \frac{1}{\left|1+\alpha \beta e^{-\mu \lambda}\right|-\left(|\alpha+\beta|+\frac{\frac{\mu}{2}|\alpha-\beta|}{\sqrt{\delta-\frac{\mu^{2}}{4}}}\right) e^{-\frac{\mu \lambda}{2}}}
$$

Lemma 3. ([2]) Suppose that $\varphi, \psi \in D(A), \xi \in D\left(A^{\frac{1}{2}}\right)$ and $f(t)$ is continuously differentiable on $[0,1]$. Then there is unique solution of problem 
(1) and the following formulas hold:

$$
\begin{aligned}
& u(t)=\frac{1}{1-\gamma}\left\{\varphi+\gamma\left(B+\frac{\mu^{2}}{4}\right)^{-1}\right. \\
& \times\left(\mu-e^{-\frac{\mu}{2} \lambda}\left(\mu C(\lambda)-\left(\frac{\mu^{2}}{4}-B\right) S(\lambda)\right)\right)\left[\psi+\alpha u^{\prime}(\lambda)\right] \\
& +\left[\left(B+\frac{\mu^{2}}{4}\right)^{-1}\left(I-e^{-\frac{\mu}{2} \lambda}\left(\frac{\mu}{2} S(\lambda)+C(\lambda)\right)\right)\right] \\
& \times\left[\xi+\beta u^{\prime \prime}(\lambda)\right]+\left(B+\frac{\mu^{2}}{4}\right)^{-1} \\
& \left.\times \int_{0}^{\lambda}\left(I-e^{-\frac{\mu}{2}(\lambda-s)}\left(\frac{\mu}{2} S(\lambda-s)+C(\lambda-s)\right)\right) f(s) d s\right\} \\
& +\left(B+\frac{\mu^{2}}{4}\right)^{-1}\left(\mu-e^{-\frac{\mu}{2} t}\left(\mu C(t)-\left(\frac{\mu^{2}}{4}-B\right) S(t)\right)\right) \\
& \times\left[\psi+\alpha u^{\prime}(\lambda)\right]+\left(B+\frac{\mu^{2}}{4}\right)^{-1}\left(I-e^{-\frac{\mu}{2} t}\left(\frac{\mu}{2} S(t)+C(t)\right)\right) \\
& \times\left[\xi+\beta u^{\prime \prime}(\lambda)\right]+\left(B+\frac{\mu^{2}}{4}\right)^{-1} \\
& \times \int_{0}^{t}\left(I-e^{-\frac{\mu}{2}(t-s)}\left(C(t-s)+\frac{\mu}{2} S(t-s)\right)\right) f(s) d s, \\
& u^{\prime}(\lambda)=P\left\{\left\{e^{-\frac{\mu}{2} \lambda}\left[C(\lambda)+\frac{\mu}{2} S(\lambda)\right]-\beta e^{-\mu \lambda} I\right\} \psi\right. \\
& +\left\{e^{-\frac{\mu}{2} \lambda} S(\lambda)-\beta \mu e^{-\mu \lambda} S^{2}(\lambda)\right\} \xi-\beta e^{-\frac{\mu}{2} \lambda} \\
& \left.\times \int_{0}^{\lambda} e^{-\frac{\mu}{2}(\lambda-s)}(S(\lambda) C(\lambda-s)-C(\lambda) S(\lambda-s)) f(s) d s\right\}, \\
& u^{\prime \prime}(\lambda)=P\left\{\left\{-e^{-\frac{\mu}{2} \lambda} S(\lambda)+\alpha \mu e^{-\mu \lambda} S^{2}(\lambda)\right\}\right. \\
& \times\left(\frac{\mu^{2}}{4}+B\right) \psi+\left\{e^{-\frac{\mu}{2} \lambda}\left(C(\lambda)-\frac{\mu}{2} S(\lambda)\right)-\alpha e^{-\mu \lambda} I\right\} \xi \\
& +\int_{0}^{\lambda}\left(-\frac{\mu}{2} S(\lambda-s)+C(\lambda-s)\right) e^{-\frac{\mu}{2}(\lambda-s)} f(s) d s \\
& \left.-\alpha \int_{0}^{\lambda} e^{-\frac{\mu}{2}(2 \lambda-s)}\left(C(s)-\frac{\mu}{2} S(s)\right) f(s) d s\right\} \text {. }
\end{aligned}
$$

Now we will formulate our main theorem.

Theorem 4. Suppose that $\varphi \in H, \psi \in D(B), \xi \in D\left(B^{1 / 2}\right)$ and $f(t)$ is continuous on $[0,1]$ and $f(t) \in D\left(B^{1 / 2}\right)$. Then there is a unique solution of 
problem (1) and the following inequalities hold:

$$
\begin{gathered}
\max _{0 \leq t \leq 1}\|u(t)\|_{H} \leq M(\gamma, \alpha, \beta, \delta)\left[\|\varphi\|_{H}+\left\|B^{-\frac{1}{2}} \psi\right\|_{H}\right. \\
\left.+\left\|B^{-1} \xi\right\|_{H}+\max _{0 \leq t \leq 1}\left\|B^{-1} f(t)\right\|_{H}\right], \\
\max _{0 \leq t \leq 1}\left\|\frac{d^{3} u(t)}{d t^{3}}\right\|_{H}+\max _{0 \leq t \leq 1}\left\|\mu \frac{d^{2} u(t)}{d t^{2}}\right\|_{H}+\max _{0 \leq t \leq 1}\left\|B \frac{d u(t)}{d t}\right\|_{H} \\
\leq M(\alpha, \beta, \delta)\left[\|B \psi\|_{H}+\left\|B^{\frac{1}{2} \xi}\right\|_{H}+\max _{0 \leq t \leq 1}\left\|B^{\frac{1}{2}} f(t)\right\|_{H}\right],
\end{gathered}
$$

where $M(\gamma, \alpha, \beta, \delta), M(\alpha, \beta, \delta)$ do not depend on $f(t), \varphi, \psi, \xi$.

Proof. First, we estimate $\|u(t)\|_{H}$ for $t \in[0,1]$. Applying (7), triangle inequality and estimates (4), we get

$$
\begin{aligned}
& \|u(t)\|_{H} \leq \frac{1}{|1-\gamma|}\left\{\|\varphi\|_{H}+|\gamma| M(\delta)\left[\left\|B^{-\frac{1}{2}} \psi\right\|_{H}+|\alpha|\left\|B^{-\frac{1}{2}} u^{\prime}(\lambda)\right\|_{H}\right]\right. \\
& \left.+M(\delta)\left[\left\|B^{-1} \xi\right\|_{H}+|\beta|\left\|B^{-1} u^{\prime \prime}(\lambda)\right\|_{H}\right]+M(\delta) \max _{0 \leq t \leq 1}\left\|B^{-1} f(t)\right\|_{H}\right\}
\end{aligned}
$$

for any $t \in[0,1]$. Then, the proof of estimate (10) is based on the inequalities

$$
\begin{gathered}
\left\|B^{-\frac{1}{2}} u^{\prime}(\lambda)\right\|_{H} \leq M\left\{(1+|\beta|)\left\|B^{-\frac{1}{2}} f_{1}\right\|_{H}+|\beta|\left\|B^{-1} f_{2}\right\|_{H}\right\}, \\
\left\|B^{-1} u^{\prime \prime}(\lambda)\right\|_{H} \leq M\left\{(1+|\alpha|)\left\|B^{-1} f_{2}\right\|_{H \rightarrow H}+|\alpha|\left\|B^{-\frac{1}{2}} f_{1}\right\|_{H}\right\} \\
\left\|B^{-\frac{1}{2}} f_{1}\right\|_{H} \leq M(\delta)\left\{\left|1+\frac{\mu}{2}\right|\left\|B^{-\frac{1}{2}} \psi\right\|_{H}+\left\|B^{-1} \xi\right\|_{H}\right. \\
\left.+\max _{0 \leq t \leq 1}\left\|B^{-1} f(t)\right\|_{H}\right\}, \\
\left\|B^{-1} f_{2}\right\|_{H} \leq M(\delta)\left\{\left\|B^{-\frac{1}{2}} \psi\right\|_{H}+\left|1+\frac{\mu}{2}\right|\left\|B^{-1} \xi\right\|_{H}\right. \\
\left.+\max _{0 \leq t \leq 1}\left\|B^{-1} f(t)\right\|_{H}\right\} .
\end{gathered}
$$

Second, we estimate $\left\|B \frac{d u(t)}{d t}\right\|_{H}$ for $t \in[0,1]$. Applying (7) and taking derivative, we get

$$
\begin{aligned}
& \left\|B \frac{d u(t)}{d t}\right\|_{H} \leq M\left\{\left|1+\frac{\mu}{2}\right|\left(|\alpha|\left\|B u^{\prime}(\lambda)\right\|_{H}+\|B \psi\|_{H}\right)\right. \\
& \left.+|\beta|\left\|B^{\frac{1}{2}} u^{\prime \prime}(\lambda)\right\|_{H}+\left\|B^{\frac{1}{2}} \xi\right\|_{H}+\max _{0 \leq t \leq 1}\left\|B^{\frac{1}{2}} f(t)\right\|_{H}\right\}
\end{aligned}
$$


for any $t \in[0,1]$. Applying formulas (8), (9), using the triangle inequality and estimates (4), we get

$$
\begin{gathered}
\left\|B u^{\prime}(\lambda)\right\|_{H} \leq M(\delta)\left\{(1+|\beta|)\left\|B f_{1}\right\|_{H}+|\beta|\left\|B^{\frac{1}{2}} f_{2}\right\|_{H}\right\} \\
\left\|B^{\frac{1}{2}} u^{\prime \prime}(\lambda)\right\|_{H} \leq M(\delta)\left\{|\alpha|\left\|B f_{1}\right\|_{H}+(1+|\alpha|)\left\|B^{\frac{1}{2}} f_{2}\right\|_{H}\right\}, \\
\left\|B f_{1}\right\|_{H} \leq M(\delta, \mu)\left\{\|B \psi\|_{H}+\left\|B^{\frac{1}{2}} \xi\right\|_{H}\right. \\
\left.+\max _{0 \leq t \leq 1}\left\|B^{\frac{1}{2}} f(t)\right\|_{H}\right\} \\
\left\|B^{\frac{1}{2}} f_{2}\right\|_{H} \leq M(\delta, \mu)\left\{\|B \psi\|_{H}+\left\|B^{\frac{1}{2}} \xi\right\|_{H}\right. \\
\left.+\max _{0 \leq t \leq 1}\left\|B^{\frac{1}{2}} f(t)\right\|_{H}\right\} .
\end{gathered}
$$

Combining estimates (16), (17), (18) and (19), we obtain estimate

$$
\left\|B \frac{d u(t)}{d t}\right\|_{H} \leq M\left\{\|B \psi\|_{H}+\left\|B^{\frac{1}{2}} \xi\right\|_{H}+\max _{0 \leq t \leq 1}\left\|B^{\frac{1}{2}} f(t)\right\|_{H}\right\} .
$$

Now, we estimate $\left\|\mu \frac{d^{2} u}{d t^{2}}\right\|_{H}$ for $t \in[0,1]$. Applying (7) and taking derivatives, we get

$$
\begin{aligned}
& \left\|\mu \frac{d^{2} u}{d t^{2}}\right\|_{H} \leq M(\delta)\left\{\mu\left(|\alpha|\left\|B u^{\prime}(\lambda)\right\|_{H}+\|B \psi\|_{H}\right)\right. \\
& +\left(1+\frac{\mu}{2}\right)\left(|\beta \mu|\left\|B^{\frac{1}{2}} u^{\prime \prime}(\lambda)\right\|_{H}+\left\|B^{\frac{1}{2}} \xi\right\|_{H}\right) \\
& \left.+\max _{0 \leq t \leq 1}\left\|B^{\frac{1}{2}} f(t)\right\|_{H}\right\} .
\end{aligned}
$$

Combining estimates (16), (17), (18) and (19), we obtain estimate

$$
\left\|\mu \frac{d^{2} u}{d t^{2}}\right\|_{H} \leq M\left\{\|B \psi\|_{H}+\left\|B^{\frac{1}{2}} \xi\right\|_{H}+\max _{0 \leq t \leq 1}\left\|B^{\frac{1}{2}} f(t)\right\|_{H}\right\} .
$$

From that and equation (1) and triangle inequality, it follows that

$$
\max _{0 \leq t \leq 1}\left\|\frac{d^{3} u(t)}{d t^{3}}\right\|_{H} \leq M\left\{\|B \psi\|_{H}+\left\|B^{\frac{1}{2}} \xi\right\|_{H}+\max _{0 \leq t \leq 1}\left\|B^{\frac{1}{2}} f(t)\right\|_{H}\right\} .
$$

The proof of Theorem 4 is finished. 


\section{Applications}

In this section we will consider two applications of main Theorem 4. First, we consider the nonlocal boundary value problem for a third order partial differential equation

$$
\left\{\begin{array}{l}
\frac{\partial^{3} u(t, x)}{\partial t^{3}}+\mu \frac{\partial^{2} u(t, x)}{\partial t^{2}}-\left(a(x) u_{t x}\right)_{x} \\
\quad+\delta u_{t}(t, x)=f(t, x), \quad 0<t<1,0<x<l \\
u(0, x)=\gamma u(\lambda, x)+\varphi(x), u_{t}(0, x)=\alpha u_{t}(\lambda, x)+\psi(x) \\
u_{t t}(0, x)=\beta u_{t t}(\lambda, x)+\xi(x), \quad 0 \leq x \leq l, 0<\lambda \leq 1 \\
u(t, 0)=u(t, l), \quad u_{x}(t, 0)=u_{x}(t, l), \quad 0 \leq t \leq 1
\end{array}\right.
$$

Problem (20) has a unique smooth solution $u(t, x)$ for smooth $a(x) \geq a>0$, $x \in(0, l), \delta>0, a(l)=a(0), \varphi(x), \psi(x), \xi(x)(x \in[0, l])$ and $f(t, x)(t \in$ $(0,1), x \in(0, l))$ functions and all compatibility conditions are satisfied.

Let us give the stability results.

Theorem 5. For the solution of the problem (20), the stability inequalities

$$
\begin{aligned}
& \max _{0 \leq t \leq 1}\|u(t, .)\|_{L_{2}[0,1]} \leq M_{1}\left[\max _{0 \leq t \leq 1}\|f(t, .)\|_{L_{2}[0,1]}\right. \\
& \left.+\|\varphi\|_{L_{2}[0,1]}+\|\psi\|_{L_{2}[0,1]}+\|\xi\|_{L_{2}[0,1]}\right] \text {, } \\
& \max _{0 \leq t \leq 1}\left\|\frac{\partial u}{\partial t}(t, .)\right\|_{W_{2}^{2}[0,1]}+\max _{0 \leq t \leq 1}\left\|\frac{\partial^{3} u}{\partial t^{3}}(t, .)\right\|_{L_{2}[0,1]} \\
& +\max _{0 \leq t \leq 1}\left\|\mu \frac{\partial^{2} u}{\partial t^{2}}(t, .)\right\|_{L_{2}[0,1]} \\
& \leq M_{1}\left[\max _{0 \leq t \leq 1}\|f(t, .)\|_{W_{2}^{1}[0,1]}+\|\psi\|_{W_{2}^{2}[0,1]}+\|\xi\|_{W_{2}^{1}[0,1]}\right]
\end{aligned}
$$

hold, where $M_{1}$ does not depend on $f(t, x)$ and $\varphi(x), \psi(x), \xi(x)$.

Proof. Problem (20) can be written as abstract problem (1) in Hilbert space $H=L_{2}[0, l]$ for all square integrable functions defined on $[0, l]$ with self-adjoint positive definite operator $A=A^{x}$ defined by the formula

$$
A^{x} u(x)=-\left(a(x) u_{x}\right)_{x}+\delta u(x)
$$

with domain

$$
\begin{gathered}
D\left(A^{x}\right)=\left\{u(x): u, u_{x},\left(a(x) u_{x}\right)_{x} \in L_{2}[0, l]\right. \\
\left.u(0)=u(l), u^{\prime}(0)=u^{\prime}(l)\right\}
\end{gathered}
$$


Here $f(t)=f(t, x)$ and $u(t)=u(t, x)$ are respectively known and unknown abstract functions defined on $[0, l]$ with the values $L_{2}[0, l]$. Therefore, estimates (21)-(22) follow from estimates (10), (11). Thus, Theorem 5 is proved.

Second, let $\Omega \subset \mathbb{R}^{n}$ be a bounded open domain with smooth boundary $S$, $\bar{\Omega}=\Omega \cup S$. In $[0,1] \times \Omega$, we consider the boundary value problem for a third order partial differential equation

$$
\left\{\begin{array}{l}
\frac{\partial^{3} u(t, x)}{\partial t^{3}}+\mu \frac{\partial^{2} u(t, x)}{\partial t^{2}}-\sum_{r=1}^{n}\left(a_{r}(x) u_{t x_{r}}\right)_{x_{r}}=f(t, x), \\
x=\left(x_{1}, \ldots, x_{n}\right) \in \Omega, \quad 0<t<1, \\
u(0, x)=\gamma u(\lambda, x)+\varphi(x), u_{t}(0, x)=\alpha u_{t}(\lambda, x)+\psi(x), \\
u_{t t}(0, x)=\beta u_{t t}(\lambda, x)+\xi(x), \quad x \in \bar{\Omega}, \quad 0<\lambda \leq 1, \\
u(t, x)=0, \quad x \in S, \quad 0 \leq t \leq 1,
\end{array}\right.
$$

where $a_{r}(x),(x \in \Omega), \varphi(x), \psi(x), \xi(x),(x \in \bar{\Omega})$ and $f(t, x)(x \in \bar{\Omega}), x \in \Omega$ are given smooth functions and $a_{r}(x)>0$. Assume that all compatibility conditions are satisfied. Let us give the stability results.

Theorem 6. For the solution of the problem (24) the stability inequalities

$$
\begin{aligned}
& \max _{0 \leq t \leq 1}\|u(t, .)\|_{L_{2}(\bar{\Omega})} \leq M_{2}\left[\max _{0 \leq t \leq 1}\|f(t, .)\|_{L_{2}(\bar{\Omega})}\right. \\
& \left.+\|\varphi\|_{L_{2}(\bar{\Omega})}+\|\psi\|_{L_{2}(\bar{\Omega})}+\|\xi\|_{L_{2}(\bar{\Omega})}\right], \\
& \max _{0 \leq t \leq 1}\left\|\frac{\partial u}{\partial t}(t, .)\right\|_{W_{2}^{2}(\bar{\Omega})}+\max _{0 \leq t \leq 1}\left\|\frac{\partial^{3} u}{\partial t^{3}}(t, .)\right\|_{L_{2}(\bar{\Omega})} \\
& +\mu \max _{0 \leq t \leq 1}\left\|\frac{\partial^{2} u}{\partial t^{2}}(t, .)\right\|_{L_{2}(\bar{\Omega})} \\
& \leq M_{2}\left[\max _{0 \leq t \leq 1}\|f(t, .)\|_{W_{2}^{1}(\bar{\Omega})}+\|\psi\|_{W_{2}^{2}(\bar{\Omega})}+\|\xi\|_{W_{2}^{1}(\bar{\Omega})}\right]
\end{aligned}
$$

hold where $M_{2}$ does not depend on $f(t, x)$ and $\varphi(x), \psi(x), \xi(x)$.

Proof. Problem (24) can be written as abstract problem (1) in Hilbert space $H=L_{2}(\bar{\Omega})$ with self-adjoint positive definite operator $A=A^{x}$ defined by the formula

with domain

$$
A^{x} u(x)=-\sum_{r=1}^{n}\left(a_{r}(x) u_{x_{r}}\right)_{x_{r}}
$$

$$
\begin{gathered}
D\left(A^{x}\right)=\left\{u(x): u(x), u_{x_{r}}(x),\left(a_{r}(x) u_{x_{r}}\right) \in L_{2}(\bar{\Omega}),\right. \\
1 \leq r \leq n, u(x)=0, x \in S\} .
\end{gathered}
$$


Here $f(t)=f(t, x)$ and $u(t)=u(t, x)$ are known and unknown respectively abstract functions defined on $\bar{\Omega}$ with the value in $L_{2}(\bar{\Omega})$. So, estimates (25)-(26) follow from estimates (10)-(11) and from the theorem on coercivity inequality for the solution of the elliptic differential problem in $L_{2}(\bar{\Omega})[19]$. Thus, Theorem 6 is proved.

Acknowledgments: The author is grateful to Prof. Allaberen Ashyralyev for his support made this work possible and to reviewers for the useful comments and relevant references.

\section{References}

[1] A. Ashyralyev, Kh. Belakroum, A stable difference scheme for a third-order partial differential equation, Contemporary Mathematics. Fundamental Directions, 64, No 1 (2018), 1-19.

[2] A. Ashyralyev, S.N. Simsek, An operator method for a third order partial differential equation, Numer. Funct. Anal. Optim., 38, No 10 (2017), 13411359.

[3] A. Ashyralyev, Kh. Belakroum, A. Guezane-Lakoud, Stability of boundary-value problems for third-order partial differential equations, Electron. J. Differ. Equ., 2017, No 53 (2017), 1-11.

[4] A. Ashyralyev, N. Aggez and F. Hezenci, Boundary value problem for a third order partial differential equation, In: AIP Conf. Proc., 1470 (First Int. Conf. on Analysis and Applied Mathematics (ICAAM 2012), Gumushane, Turkey, Oct. 18-21, 2012) (Ed. by: A. Ashyralyev and A. Lukashov), Amer. Inst. Phys. (2012), 130-133.

[5] Yu.P. Apakov, B.Yu. Irgashev, Boundary-value problem for a degenerate high-odd-order equation, Ukrainian Math. J., 66, No 10 (2015), 1475-1490.

[6] Yu.P. Apakov, On the solution of a boundary-value problem for a thirdorder equation with multiple characteristics, Ukrainian Math. J., 64, No 1 (2012), 1-12.

[7] Yu. P. Apakov, S. Rutkauskas, On a boundary value problem to third order PDE with multiple characteristics, Nonlinear Anal. Model. Control, 16, No 3 (2011), 255-269. 
[8] A. Ashyralyev, D. Arjmand and M. Koksal, Taylor's decomposition on four points for solving third-order linear time-varying systems, J. of the Franklin Institute Engin. and Appl. Mathematics, 346, No 7 (2009), 651-662.

[9] M. Ashyraliyev, A note on the stability of the integral-differential equation of the hyperbolic type in a Hilbert space, Numer. Funct. Anal. Optim., 29, No 7-8 (2008), 750-769.

[10] A. Ashyralyev, P.E. Sobolevskii, New Difference Schemes for Partial Differential Equations, Birkhauser Verlag, Basel-Boston-Berlin (2004).

[11] Kh. Belakroum, A. Ashyralyev and A. Guezane-Lakoud, A note on the nonlocal boundary value problem for a third order partial differential equation, Filomat, 32, No 3 (2018), 801-808.

[12] Z. Direk, M. Ashyraliyev, FDM for the integral-differential equation of the hyperbolic type, Adv. Difference Equ., 2014, No 1 (2014), 1-8.

[13] H. O. Fattorini, Second Order Linear Differential Equations in Banach Spaces, Elsevier Sci. Publ. B.V, Amsterdam (1985).

[14] A. Guezane-Lakoud, N. Hamidane and R. Khaldi, On a third-order threepoint boundary value problem, Int. J. Math. Math. Sci., 2012 (2012).

[15] S.A. Gabov and A.G. Sveshnikov, Problems of the Dynamics of Stratified Fluids, Nauka, Moscow (1986) (in Russian).

[16] J. Niu, P. Li, Numerical algorithm for the third-order partial differential equation with three-point boundary value problem, Abstr. Appl. Anal., 2014 (2014).

[17] A.I. Kozhanov, Mixed boundary value problem for some classes of third order differential equations, Matematicheskii Sbornik, 118, No 160 (1982), 504-522 (in Russian).

[18] V.I. Korzyuk, V.B. Kiselev, A boundary value problem for a third-order linear nonclassical equation generated by the heat equation, Vest. Nats. Akad. Navuk Belaruser. Fiz.-Mat. Navuk, 140, No 3 (2001), 5-11.

[19] P.E. Sobolevskii, Difference Methods for the Approximate Solution of Differential Equations, Voronezh State University Press, Voronezh (1975) (in Russian). 